Revue des patrimoines

\title{
Le régiment de cavalerie de la Garde républicaine
}

\section{Chef d'escadron Frédéric Daugérias}

\section{OpenEdition}

\section{Journals}

Édition électronique

URL : http://journals.openedition.org/insitu/12160

DOI : 10.4000/insitu. 12160

ISSN : $1630-7305$

\section{Éditeur}

Ministère de la culture

\section{Référence électronique}

Chef d'escadron Frédéric Daugérias, «Le régiment de cavalerie de la Garde républicaine », In Situ [En ligne], 27 | 2015, mis en ligne le 02 novembre 2015, consulté le 19 avril 2019. URL : http:// journals.openedition.org/insitu/12160 ; DOI : 10.4000/insitu.12160

Ce document a été généré automatiquement le 19 avril 2019.

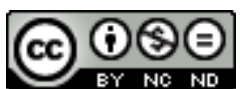

In Situ Revues des patrimoines est mis à disposition selon les termes de la licence Creative Commons Attribution - Pas d'Utilisation Commerciale - Pas de Modification 4.0 International. 


\title{
Le régiment de cavalerie de la Garde républicaine
}

\author{
Chef d'escadron Frédéric Daugérias
}

1 Le régiment de cavalerie de la Garde républicaine est la dernière unité montée des forces armées françaises qui fasse vivre et utilise son savoir-faire en matière d'équitation en arme pour effectuer des missions de sécurité publique générale.

2 En préambule, il convient de rappeler ce qu'est la garde républicaine: elle trouve ses origines dans le Guet royal créé par saint Louis au Moyen Âge. C'est au XVIII siècle qu'apparaît pour la première fois l'usage de donner au Guet royal l'appellation de " Garde de Paris ». Formé de soldats, il avait pour mission de patrouiller et d'intervenir dans la capitale.

3 La Garde évolua au gré des révolutions et des systèmes politiques français mais c'est le décret du 4 octobre 1802 (12 vendémiaire an XI) qui la créa officiellement. Elle fut rattachée à la gendarmerie en 1849 et changea de dénomination de nombreuses fois pour adopter en 1979 son organisation actuelle.

4 Les missions de la Garde sont également issues de son histoire et s'articulent autour de la dyade « Sécurité et honneur » (fig. 1). 
Figure 1

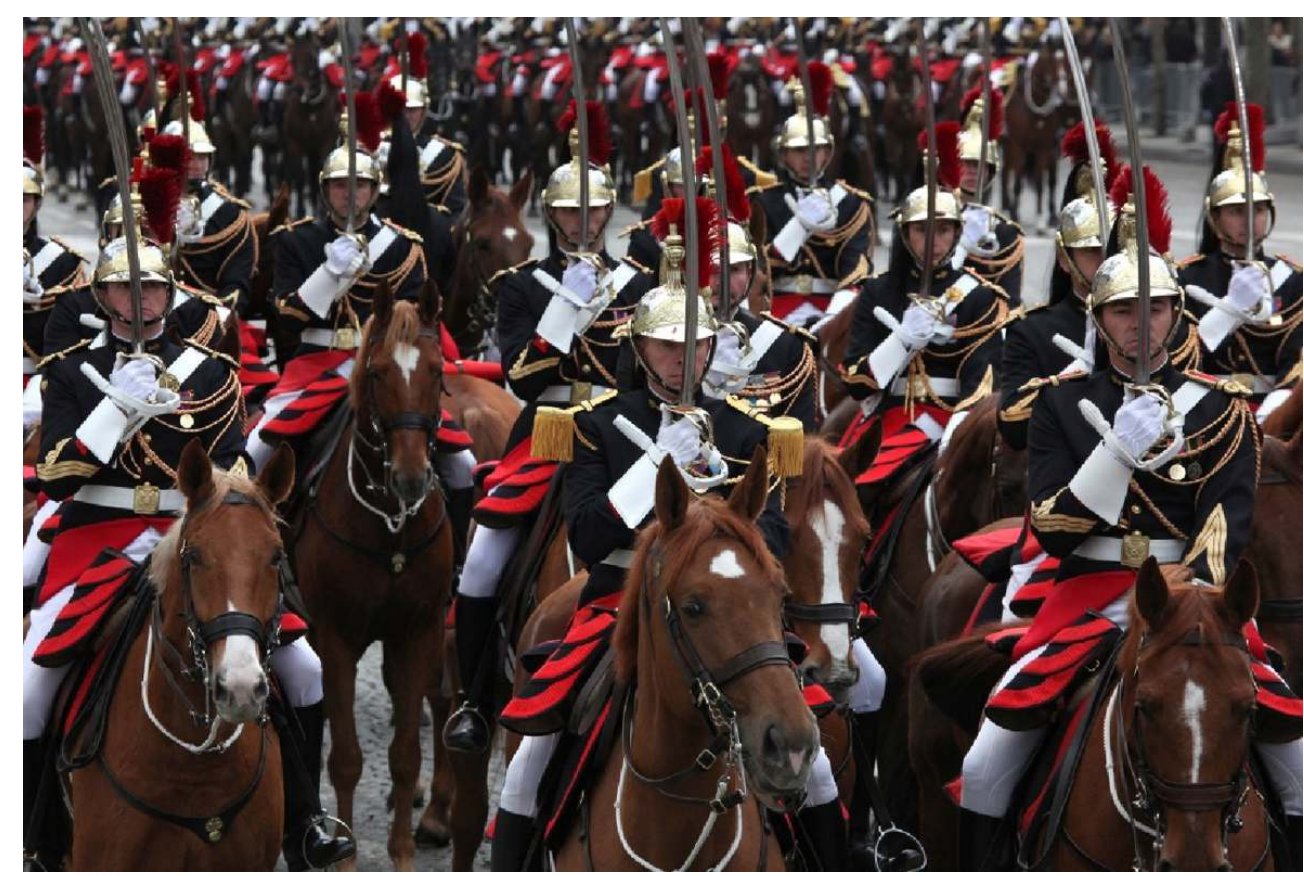

Défilé du 14 Juillet, le $1^{\text {er }}$ escadron de la Garde républicaine au « Présentez-sabre ! ».

Phot. Mendiboure, David. (c) Service communication, Garde républicaine.

5 Ainsi, le régiment de cavalerie fait partie intégrante de la Garde républicaine et donc de la gendarmerie nationale.

Les missions du régiment de cavalerie découlent de son histoire. Tout comme à l'origine le Guet royal, il a en charge les missions d'honneur et de sécurité au service du chef de l'État, des hautes autorités politiques françaises et étrangères en visite en France.

7 Ces missions se sont développées à l'extérieur de la capitale et se sont élargies à la sécurité publique générale. Le régiment est ainsi devenu la " force équestre projetable ${ }^{1}$ du ministère de l'Intérieur et intervient pour effectuer des missions de sécurité dans tout le territoire métropolitain. Le régiment de cavalerie met à disposition des autres unités de la gendarmerie nationale, mais aussi de la police, pour l'exécution de missions de sécurité ses moyens, chevaux et cavaliers.

D'autres institutions effectuent également des patrouilles à cheval : la police nationale, qui arme des brigades équestres et possède une cinquantaine de chevaux, et certaines polices municipales. Ces unités, qu'elles soient nationales ou municipales, restent toutefois rattachées à une circonscription, de même que les 7 postes à cheval du régiment de cavalerie. C'est pour cette raison qu'ils ne peuvent être mobilisés sur d'autres points du territoire.

9 Afin de bien saisir le contexte d'exécution des missions du régiment de cavalerie, il convient tout d'abord de le découvrir rapidement au travers de son organisation, pour évoquer les avantages du cheval dans les missions de sécurité publique générale et enfin, sa doctrine d'action. 


\section{L'organisation du régiment de cavalerie}

10 D'un effectif de 529 militaires et civils, le régiment de cavalerie est composé de 3 escadrons de marche, 1 escadron hors rang (de soutien) et un centre d'instruction.

11 Les trois escadrons de marche effectuent le service général du régiment de cavalerie: services d'honneur à cheval ou à pied, missions de sécurité publique.

12 Commandés par un capitaine (ou chef d'escadron), ils sont organisés en trois pelotons organiques commandés par un lieutenant ou major, et possèdent chacun une forge et un groupe de commandement. Ils comprennent chacun une centaine de cavaliers. Chaque escadron doit être en mesure d'envoyer des cavaliers pour des missions de sécurité publique et maintient pour cela en permanence 8 cavaliers et chevaux en alerte. Chaque escadron est également responsable de postes à cheval permanents qu'il fournit en chevaux et qu'il arme en personnel.

13 L'escadron de soutien comprend l'antenne vétérinaire du régiment de cavalerie (qui dépend du service de Santé des Armées), la fanfare de cavalerie et le mess. L'antenne vétérinaire est formée de 4 vétérinaires du service de santé des armées et d'auxiliaires vétérinaires qui sont d'anciens cavaliers du rang ayant reçu une formation d'infirmier vétérinaire. La fanfare de cavalerie est commandée par un capitaine trompette-major et composée de sous-officiers de gendarmerie, musiciens et cavaliers. Elle ouvre tous les défilés du régiment et les escortes à cheval, elle sonne au trot.

Le centre d'instruction forme tous les militaires et les chevaux affectés au régiment de cavalerie. Il reçoit également des stagiaires de la gendarmerie nationale (gendarmes d'active ou de réserve participant à la mise en place des postes saisonniers), des polices municipales (protocole d'accord avec le Centre national de la Fonction publique territoriale) et des stagiaires étrangers. Il dispense des formations de base, mais aussi de moniteur ou d'instructeur d'équitation.

\section{Effectif équin du régiment de cavalerie}

15 Les 461 chevaux qui le composent sont achetés en fonction de leurs qualités intrinsèques de cheval de selle, puis sont formés au travail en arme et au travail de sécurité publique. Pour diverses raisons (budget, disponibilité du personnel) l'effectif équin a été réduit sur plusieurs années.

Ce sont dans leur immense majorité des chevaux de selle français ; quelques anglo-arabes et quelques chevaux allemands ou néerlandais (KWPN, Holsteiner...) viennent compléter les rangs. Les chevaux proviennent d'élevages français, principalement normands du fait de la concentration d'éleveurs dans cette région.

17 Achetés à l'âge de 3 ans, ils doivent présenter les caractéristiques suivantes :

$18-1,65 \mathrm{~m}$ au garrot,

19 - posséder de l'os et un certain cadre,

20 - posséder de bonnes origines,

21 - avoir une robe franche,

22 - faire déjà preuve d'un caractère facile. 
23 Aussitôt sélectionnés, ils rejoignent l'antenne vétérinaire où ils subissent de nombreux tests dynamiques et statiques, dont 16 radiographies des membres. Si ces tests sont positifs, la vente est conclue et ils rejoignent le peloton de débourrage au centre d'instruction où ils restent une année, temps nécessaire à leur débourrage et à leur accoutumance au milieu urbain. Si les tests sont négatifs, ils retournent chez leur éleveur. Ils rejoignent leur unité à l'âge de 4 ans (en fonction de la couleur de leur robe) et sont " travaillés » pendant une année, avant d'effectuer leur premier service à l'âge de 5 ans. Les chevaux sont présentés à la réforme dès l'âge de 16 ans. Certains sont réformés plus jeunes (inadaptés aux conditions d'utilisation, raison vétérinaire...), d'autres plus vieux, lorsqu'ils sont encore aptes à servir sans souffrir. Réformées, les juments peuvent être vendues à leur naisseur pour les remettre à l'élevage. Les autres chevaux sont cédés à titre gracieux aux gardes, aux particuliers qui en font la demande ou en dernier lieu, à des associations. Le transfert de propriété se fait sous condition : les chevaux ne peuvent faire l'objet d'une exploitation commerciale ou sportive, de revente, et l'acquéreur doit garantir à cet animal qui a servi la France une retraite paisible.

\section{Les missions}

26 Les missions du régiment de cavalerie sont le fruit de son histoire. Tout comme à l'origine le guet royal, il a en charge les missions d'honneur et de sécurité au profit du chef de l'État, des hautes autorités politiques françaises et étrangères en visite en France.

Ce modèle issu de la tradition médiévale est désormais un modèle original, puisque le régiment de cavalerie est une des seules unités à assurer à la fois des services d'honneur à pied, à cheval et à participer activement à la sécurité publique générale et à l'ordre public. Pour Napoléon $\mathrm{I}^{\mathrm{er}}$, « une troupe chargée de maintenir l'ordre au dedans ne doit pas être privée de l'honneur de servir la grandeur de la Patrie au dehors. Elle ne reviendra que meilleure et plus respectée ${ }^{2}$.»

Cette dualité résultant de l'histoire de la garde républicaine est aussi la particularité de la gendarmerie nationale française : unité militaire à part entière, elle rend les honneurs militaires et effectue des missions civiles de police.

Fondées sur ce principe, les missions de la Garde républicaine en général et du régiment de cavalerie en particulier se sont déplacées à l'extérieur de la capitale et ont évolué vers la sécurité publique générale.

Dernière unité de cavalerie montée des forces armées françaises, le régiment de cavalerie est devenu à la fois :

31 - la force équestre projetable du ministère de l'Intérieur, effectuant des missions de sécurité dans tout le territoire métropolitain, une unité de prestige qui participe au premier rang au protocole de l'État,

32 - le conservatoire des traditions équestres françaises.

33 Honneur et sécurité sont les deux missions de la Garde républicaine et du régiment de cavalerie. Si la mission d'honneur est la plus emblématique, elle ne représente que $30 \%$ de l'activité du régiment contre $70 \%$ pour les missions de sécurité publique.

La mission qui a le plus d'intérêt ici est celle de sécurité publique. 


\section{Les postes à cheval permanents} (fig. 2)

Figure 2

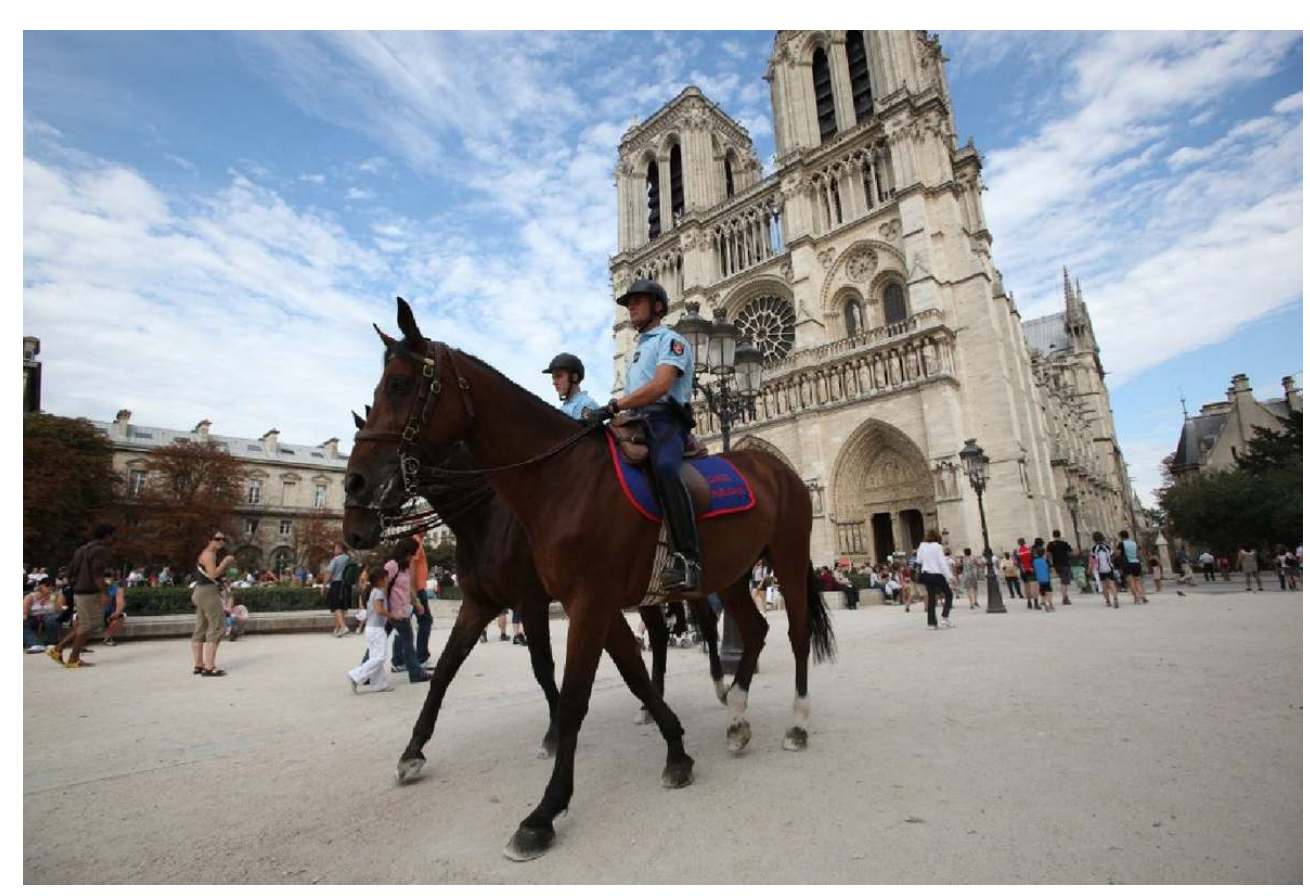

Patrouille de sécurité publique générale dans Paris intra-muros.

Phot. Mendiboure, David. (C) Service communication, Garde républicaine.

Ils comprennent les postes d'Achères, Chambord, Chantilly, L'Isle-Adam, Saint-Chéron, Saint-Nom-La-Bretèche, du bois de Boulogne et du bois de Vincennes.

- PSIC (Peloton de Surveillance et d'Intervention à Cheval).Népoui (Nouvelle Calédonie) : contact avec les villages éloignés, environnement, stupéfiants, braconnage.

Les cavaliers apportent les capacités de leur «moyen » spécial (le cheval) pour exercer la surveillance d'une zone particulière (en général massif forestier). Ils apportent également leur concours par leur savoir, orienté le plus souvent vers les atteintes à l'environnement. Les infractions les plus souvent relevées concernent ces dernières et les atteintes à la tranquillité publique, mais aussi le travail illégal, le braconnage ou les stupéfiants (plantations de cannabis).

Ces missions étaient auparavant essentiellement exercées dans les zones boisées ou vertes. Elles se tournent désormais résolument vers les zones urbaines, pour prévenir essentiellement les délits. 8000 à 9000 patrouilles sont effectuées chaque année.

- Les postes à cheval des bois de Boulogne et Vincennes

Les cavaliers assurent des patrouilles de surveillance générale au profit de la police dans les bois de Boulogne et Vincennes et à Paris intra-muros : Tuileries, Champs de Mars...

Près de 5000 patrouilles par an sont effectuées. Ce sont essentiellement des zones fortement fréquentées par les touristes ou par des familles. L'objectif est de lutter contre les atteintes à la tranquillité publique, et les malfaiteurs (vols à l'arraché, pickpockets) et autres trafics. 

principalement autour des deux grands stades parisiens (parc des Princes et stade de France), à raison de plus de cinquante services annuels. Ils ont pour mission de renseigner l'autorité civile le plus loin possible en amont, à l'arrivée des supporters, là où il n'y a pas de caméras de surveillance. Il s'agit également, au stade de France, de scinder la foule et de réguler les mouvements de personnes vers les moyens de transport. effectifs varient en fonction de l'événement (concert, match de football) et des risques possibles (en fonction de la dangerosité des supporters notamment). 6 à 18 cavaliers (une à trois escouades) sont sur place.

\section{Les grands rassemblements de personnes}

Les cavaliers du régiment de cavalerie participent aux dispositifs mis en place lors d'événements particuliers et anticipés (grands événements de type G8-G20, sommets de chefs d'État, grandes commémorations, venues du pape à Paris ou à Lourdes...). Le détachement est alors placé sous les ordres du commandant du groupement opérationnel. Les effectifs engagés vont de 12 à 80 chevaux. 

de l'événement, le contrôle des personnes, ou bien encore le respect de la réglementation particulière mise en place à cette occasion (interdiction de circulation ou d'accès à une zone).

50 Ils servent également à ouvrir les passages de véhicules officiels ou de secours à travers la foule, notamment lors de foires qui rassemblent 30 à 40000 personnes.

\section{Les opérations particulières}

51 Les cavaliers renforcent les unités territoriales lors d'opérations particulières destinées à prévenir un certain type d'infraction. À titre d'exemple, il s'agit notamment de la surveillance des vendanges (fig. 3) en Champagne (prévention des vols de raisin, du travail illégal) pendant la saison mais aussi en amont et en aval, ou bien encore, de la surveillance des parcs ostréicoles en fin d'année.

Figure 3

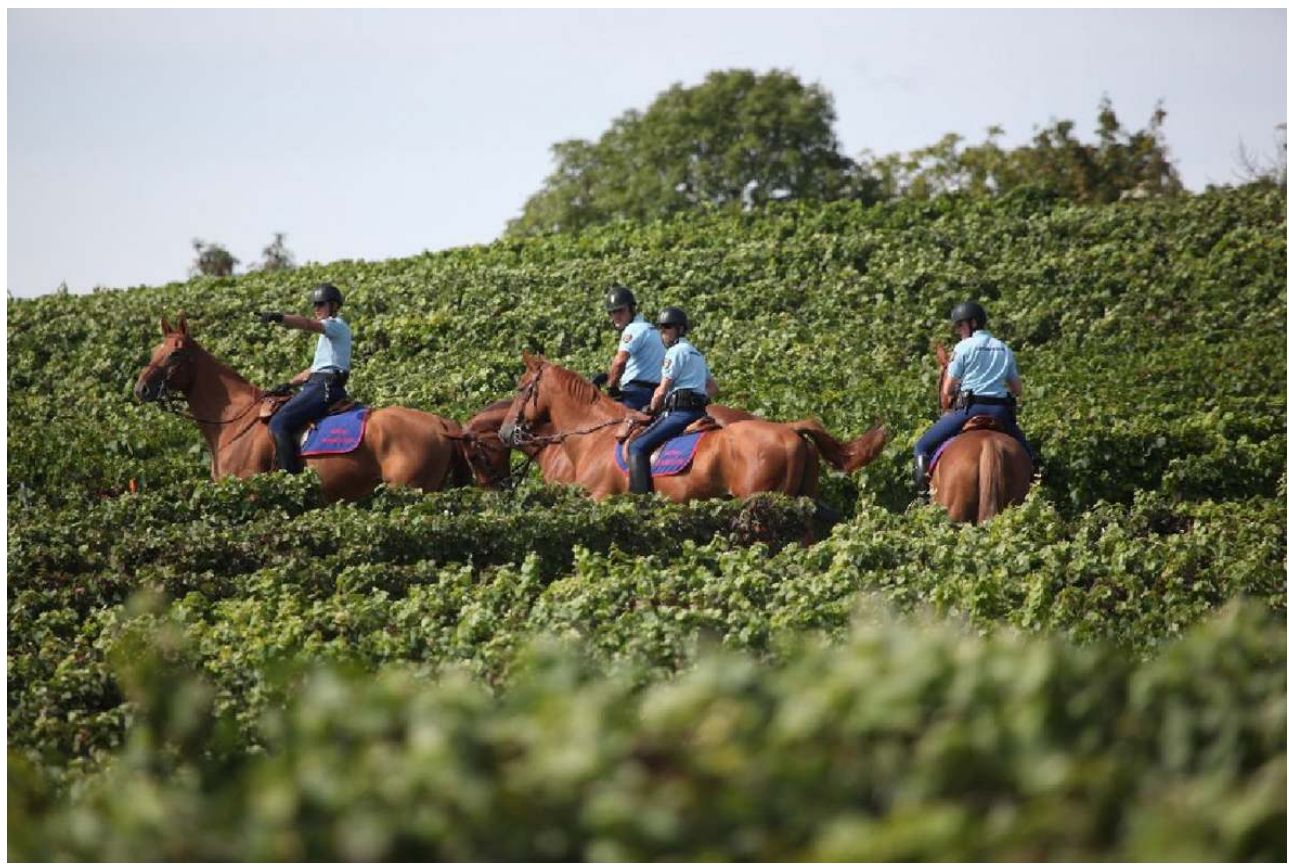

Patrouille de sécurité publique générale lors des vendanges en Champagne. « Voir loin et être vu de loin.. ».

Phot. Mendiboure, David. (c) Service communication, Garde républicaine.

Les effectifs engagés vont de 6 à 18 chevaux.

Les cavaliers du régiment de cavalerie doivent ainsi être très polyvalents : ils peuvent un jour faire un service d'honneur, un autre travailler à cheval, ou encore renforcer la gendarmerie départementale à l'autre bout du territoire. 


\section{Les avantages du cheval dans les missions de surveillance et de sécurité}

54 Avec un dressage fondé sur l'accoutumance progressive à un contexte particulier (agitation, bruit, fumée), le cheval peut évoluer dans un environnement qui lui semble a priori peu naturel (foule, ville, musique, lumière artificielle, fumigènes...).

L'intérêt du cheval dans les missions de sécurité publique générale : sa souplesse et sa mobilité. Le cheval est mobile, rapide, toujours en mouvement et à l'écoute. Son cavalier doit rester lui-même, toujours actif. Le cheval réagit de manière naturelle aux événements susceptibles d'attirer l'attention du cavalier sur certains dangers.

La position dominante du cavalier : la position élevée du cavalier (regard à environ $3 \mathrm{~m} \mathrm{du}$ sol) permet de voir et d'être vu. À cheval, le regard est omnidirectionnel. Cette position convient parfaitement à une évolution dans un sous-bois, sur un terrain accidenté ou au milieu d'une foule. Le cavalier peut évaluer au plus loin la physionomie d'une situation. Grâce au cheval, le cavalier effectue une surveillance constante car, dégagé du souci de son propre déplacement, il peut se consacrer à sa mission d'observation.

Visibilité pour le public : la position élevée du cavalier le rend visible de loin et favorise dès lors la prévention de troubles à l'ordre public. En outre, dans la pénombre, le public aperçoit toujours de loin un dispositif à cheval, contrairement à un dispositif à pied.

Effet de masse sur la foule : la capacité de dissuasion du cheval est un atout manifeste. Grâce à leur position en hauteur, les cavaliers provoquent un impact psychologique d'autant plus fort qu'il a affaire à un public citadin, connaissant peu ou mal les animaux. Cet effet de distanciation, très efficace, est lié à l'impression produite sur le piéton par la grandeur du cheval. Ainsi, pour s'adresser au cavalier, le piéton est tenu de se tenir à distance s'il veut croiser son regard.

Économie en personnel : une unité constituée (l'unité tactique de base étant l'escouade de 6 cavaliers), les gendarmes à cheval, positionnés en barrage de canalisation ou filtrant, de front ou de profil, permettent d'économiser l'équivalent de deux, voire trois pelotons de forces de l'ordre, pour une même mission. Schématiquement, un cavalier et son cheval couvrent une longueur de 2,5 mètres et une largeur d'1 mètre. Une escouade, positionnée de face, présente un front de 6 mètres et, en colonne, une largeur de 15 mètres, soit la valeur d'un peloton de gendarmes à pied.

De cet intérêt découlent deux modes d'action : la mobilité, le cheval étant instinctivement en mouvement, cette inclination doit se traduire dans son emploi en sécurité publique par le principe de la mobilité, et l'appui des troupes à pied, la patrouille doit pouvoir compter sur le renfort éventuel de personnels à pied ou motorisés. Pour un service d'ordre, le dispositif mixte de type « cavalerie-infanterie » est de rigueur.

\section{La doctrine d'action et la formation}

61 L'action du régiment de cavalerie est guidée par une doctrine et une formation de son personnel qui lui sont propres. Ancré sur des principes ancestraux, il sait s'adapter aux situations nouvelles qu'il rencontre et est en constante évolution. (L'action du régiment de cavalerie repose sur des principes anciens et sur les règlements de la cavalerie tels qu'ils ont été posés à la fin du XIX ${ }^{\mathrm{e}}$ siècle et au début $\mathrm{du} \mathrm{XX}^{\mathrm{e}}$ siècle). 
62 La dernière unité montée des forces armées françaises est à ce titre dépositaire de l'héritage de l'équitation militaire et plus spécifiquement, de l'équitation en arme de tradition française dont elle conserve la pratique vivante dans le cadre de son service quotidien, que ce soit en service d'honneur ou en mission de sécurité publique.

63 Ainsi, on passe de l'école d'escouade traditionnelle telle qu'elle était présentée dans les précis militaires successifs et notamment celui, fondateur, de la cavalerie moderne de 1875, à l'école d'intervention professionnelle à cheval utilisée de nos jours, l'unique fil conducteur étant l'unicité du couple cavalier-cheval.

64 La doctrine d'action que suit le régiment de cavalerie trouve sa source dans ces règlements, mais aussi dans la doctrine d'action des forces mobiles de la gendarmerie. Elle est également en constante évolution, car elle doit s'adapter aux modes d'action des différents adversaires rencontrés par les cavaliers.

Les chevaux sont débourrés actuellement selon une méthode qui repose sur le comportement des chevaux (méthode Blondeau).

66 Après s'être familiarisé à porter le cavalier sur son dos, le poulain est amené à passer de nombreuses embûches en main, puis monté. Ces parcours sont les prémices aux « obstacles » que le cheval pourra rencontrer au cours de sa carrière et notamment, dans ses missions de sécurité publique générale et de service d'ordre (fig. 4).

Figure 4

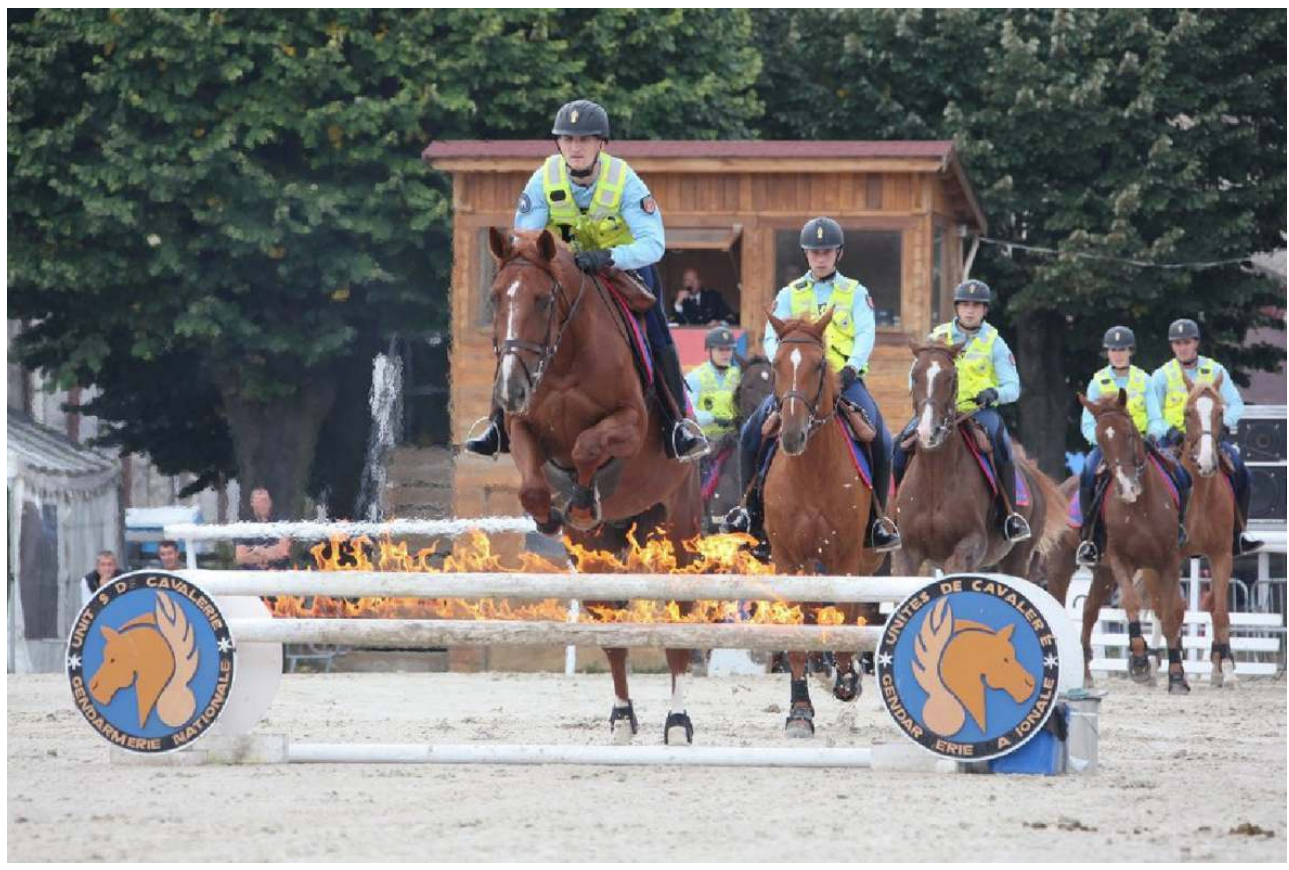

Franchissement d'obstacle en feu.

Phot. Mendiboure, David. (c) Service communication, Garde républicaine.

67 Par la suite, lorsqu'il sera en unité, ce travail sera poursuivi et entretenu.

68 Avant chaque service d'ordre, où les chevaux risquent le plus d'être confrontés à ce genre de situation, un travail spécifique est organisé pour leur rappeler ces principes de bases. Parallèlement à cela, le cheval est également "travaillé » en arme. Il apprend la selle d'arme, la bride d'arme, le sabre et le maniement du sabre. Il apprend ensuite à évoluer 
en unité constituée et s'initie au travail d'escouade, aux patrouilles mobiles et aux dispositifs statiques. La dualité des chevaux du régiment de cavalerie, qui doivent être à la fois des chevaux d'honneur et de sécurité publique, est ainsi acquise grâce à cette double formation équestre et militaire.

« L'équitation en $\mathrm{arme}^{3}$, l'authentique réponse à un besoin opérationnel toujours présent.

La cavalerie moderne naît d'un besoin opérationnel nouveau qui implique, pour trouver une réponse satisfaisante, un processus de maturation historique se traduisant par une rationalisation dans l'utilisation du cheval à la guerre (fig. 5). Cette nécessaire rationalisation a pour but d'adapter l'instruction des cavaliers et des chevaux disponibles afin de les rendre efficaces face à l'adversaire.

Figure 5

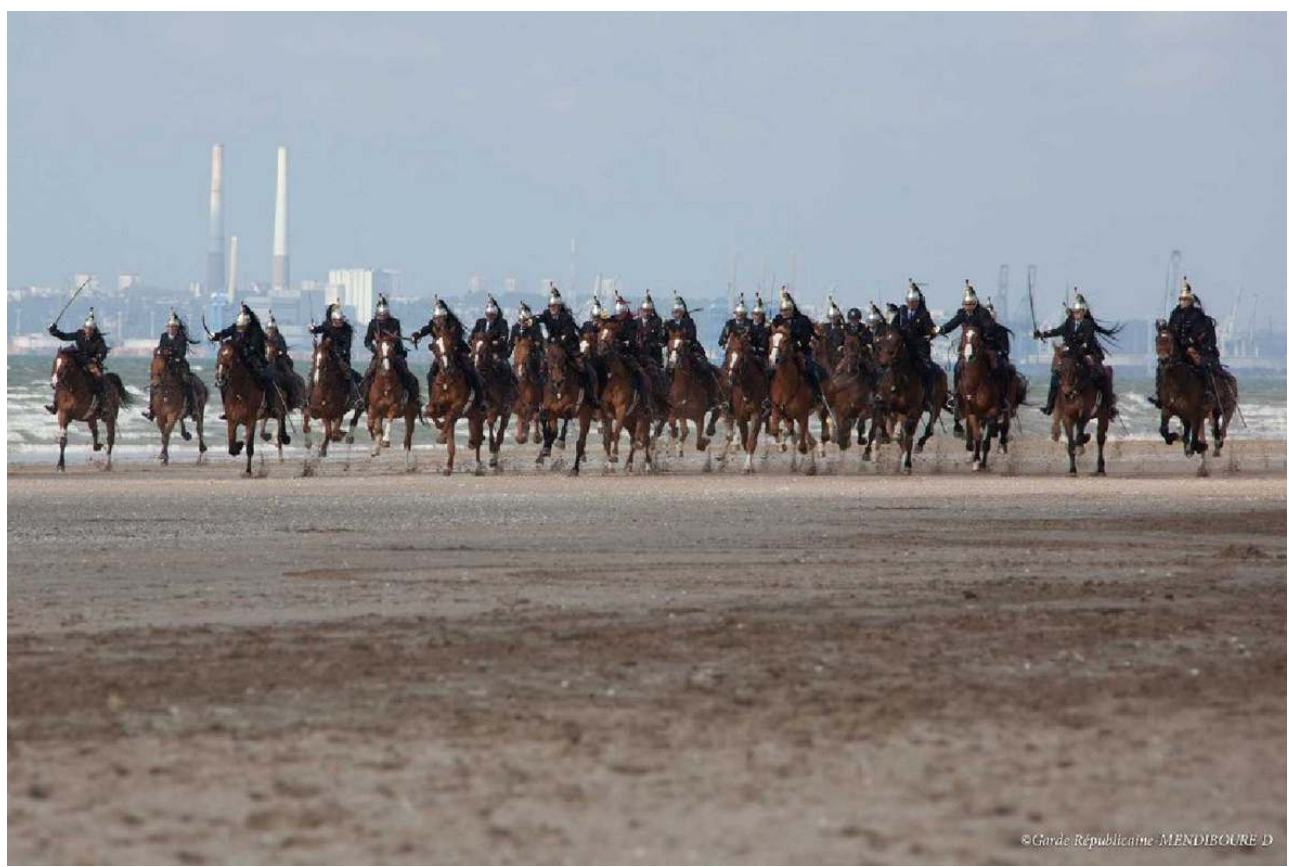

EXERCICE de CHARge À deAUVILLE, MAI 2014.

Phot. Mendiboure, David. (C) Service communication, Garde républicaine.

s'affiner les pratiques utilitaires, dans tous les domaines ayant trait au fonctionnement de la cavalerie, de la maréchalerie aux vétérinaires en passant par les artisans bourreliers, armuriers, casquiers... pour concourir à fonder ce qui s'appellera l'équitation militaire en arme. Des centaines de milliers de chevaux et de cavaliers seront formés en France à cette école et feront montre de leur esprit cavalier, tant dans les circonstances tragiques des conflits que dans tous les domaines de la vie équestre.

Aujourd'hui, seul le régiment de cavalerie de la Garde républicaine maintient cette tradition et continue de transmettre l'enseignement de rigueur et de rusticité de l'équitation en arme de tradition française. En outre, il fait vivre cette technique et cette culture d'homme de cheval au quotidien dans son fonctionnement comme dans tous ses services, aussi bien pour les honneurs que dans les missions de sécurité publique. 
74 Ainsi [...], l'équitation en arme sait encore de nos jours répondre aux nécessités de l'utilisation opérationnelle de chevaux en sécurité publique. Elle véhicule en outre des valeurs exigeantes de militarité et de professionnalisme qui font du régiment de cavalerie une unité respectée au niveau international pour son expertise et sa polyvalence dans le domaine de la formation, de l'utilisation du cheval d'arme et de son soutien au sein d'une grande unité.»

\section{NOTES}

1. - « La force équestre projetable » est l'intitulé du ministère de l'Intérieur en 2011 pour assurer la communication du régiment de cavalerie. Voir le site: http://mobile.interieur.gouv.fr/ Actualites/L-actu-du-Ministere/Les-unites-equestres-de-la-police-nationale-et-la-Garde-

Republicaine-au-salon-du-cheval.

2. - Déclaration de Napoléon $\mathrm{I}^{\mathrm{er}}$ au roi de Naples. "La gendarmerie, les gendarmes et la guerre». Lettre du 16 mai 1806, société nationale de l'histoire et du patrimoine de la gendarmerie, février 2006.

3. - D'après le travail de recherche du capitaine Gateau-Leblanc, commandant le $1^{\mathrm{er}}$ peloton du $2^{\mathrm{e}}$ escadron du régiment de cavalerie, Histoire générale de l'équitation en arme.

\section{RÉSUMÉS}

Le régiment de cavalerie de la Garde républicaine fait partie intégrante de la gendarmerie nationale. Unité multiséculaire, forte de plus de 500 militaires et civils et de quelques 460 chevaux, les missions du régiment de cavalerie sont le fruit de son histoire et tournent autour des honneurs aux plus hautes autorités de l'État ainsi que la sécurité des biens et des personnes. $\mathrm{Au}$ fil des siècles, ces missions ont évolué ; depuis l'école d'escouade des précis militaires de la cavalerie moderne de 1875 , on arrive aujourd'hui à l'école d'intervention professionnelle, adaptée aux risques du 21ème siècle. Cependant, rusticité et rigueur restent les maitres-mots tant dans la formation des chevaux que celle des cavaliers du régiment de cavalerie.

The cavalry regiment of the French Garde républicaine. This French cavalry regiment of the Garde républicaine is under the command of the national gendarmerie. It has about 500 horsemen and women and 460 horses. It is a unit that has a long history, stretching back over the centuries with the same tasks of honouring officials of the French State and the guests to France and carrying out various security missions. These of course have evolved considerably over the years and are now adapted to the security risks of the twenty-first century. However, in this cavalry regiment, rusticity and discipline remain the key words in the training both of the men and of the horses. 
INDEX

Keywords : French Garde républicaine, cavalry regiment, the national gendarmerie, horse, security missions

Mots-clés : Garde républicaine, régiment de cavalerie, gendarmerie nationale, Guet royal, cheval, peloton de surveillance et d'intervention à cheval, escadron, escouade, poste à cheval, débourrage, intervention professionnelle, service d'honneur, sécurité publique à cheval

\section{AUTEUR}

\section{CHEF D'ESCADRON FRÉDÉRIC DAUGÉRIAS}

commandant le 3e escadron du régiment de cavalerie de la Garde républicaine frederic.daugerias@gendarmerie.interieur.gouv.fr 